\title{
ESTUDO DA MORBIDADE EM UMA POPULAÇÃO IDOSA
}

\author{
Morbidity study in an elder population \\ Estudio de la morbilidad en la población de ancianos
}

\section{RESUMO}

Este estudo objetivou identificar os agravos que mais acometeram os idosos no período de 1998 a 2005 e a capacidade funcional dos residentes na área de abrangência de quatro unidades básicas de saúde de diferentes regiões, no Município de Cascavel, Paraná, em 2007. Os dados de morbidade foram pesquisados no Sistema de Informações Hospitalar do Sistema Único de Saúde, e foram realizadas entrevistas com 141 idosos. No período estudado, a morbidade pouco variou: as doenças do aparelho circulatório foram responsáveis pelo maior número de internamentos. A média geral de doenças referidas pelos idosos foi de 2 , com destaque para as cardiovasculares. Dentre os entrevistados, 136 (96,5\%) afirmaram realizar todas as atividades cotidianas, sem 0 auxílio de outras pessoas. 0 estudo mostra que é necessário implantar serviços voltados para as doenças crônicas, com foco na manutenção da máxima capacidade funcional pelo maior tempo possível, valorizando a autonomia e a autodeterminação dos idosos.

Palavras chave: Idoso. Morbidade. Políticas Públicas

\begin{abstract}
This study objectified to identify the injuries that affected the elders the most, between the period of 1998 to 2005 and the functional capability of the residents in the scope area of four health basic units of different regions, in the city of Cascavel, state of Paraná, in 2007. The morbidity data were researched in the hospital information system of the Health Unique System, and interviews were made with elders. In the studied period the morbidity almost didn't vary. The general average of diseases refereed by the elders was 2, with a highlight on the cardiovascular ones. Among the interviewed, 136 (96,5\%) affirmed to perform all the daily activities, without the assistance of other people. The study shows that it's necessary to implant services dedicated to the chronicle diseases, with focus in the maintenance of the maximum functional capability for as most time as it is possible, valorizing the autonomy and the auto-determination of the elders.
\end{abstract}

Keywords: Elderly. Morbidity. Public Policies.

\section{Resumen}

El objetivo del estudio fue identificar las enfermedades que más afectaron a los ancianos en el período entre 1998 y 2005 y la capacidad funcional de los residentes en el área de cobertura de cuatro unidades básicas de salud de diferentes regiones en la cuidad de Cascavel, Paraná, en 2007. Los datos de morbilidad fueron investigados en el Sistema de Información Hospitalario del Sistema Único de Salud, e se realizaron encuestas con 141 ancianos. En el periodo estudiado, la morbilidad ha variado poco, las enfermedades del sistema circulatorio fueron responsables por el mayor número de internamientos. El promedio general de las enfermedades referidas por los mayores fue de 2, con relieve para las cardiovasculares. Entre los encuestados, 136 (o sea el 96,5\%) afirmaron realizar todas las actividades cotidianas sin la ayuda de los demás. El estudio muestra la necesidad de implementar servicios dirigidos a las enfermedades crónicas, con especial atención en mantener la máxima capacidad funcional por el mayor tiempo posible, valorando la autonomía y la autodeterminación de los ancianos.

Palabras clave: Anciano. Morbilidad. Políticas Públicas.

'Universidade Estadual de Maringá/UEM. Docente do Curso de Enfermagem da Universidade Paranaense/ UNIPAR - Campus Cascavel/PR. Enfermeira da SESA/10 Regional de Saúde - Cascavel-PR. Brasil. E.mail: prabeth@terra.com.br. ²Doutora em Saúde Pública pela Fundação Osvaldo Cruz e Docente da Universidade Estadual de Maringá/UEM. Maringá-PR. Brasil. E-mail: mjscochi@uem.br, ${ }^{3}$ Enfermeira. Mestre em Enfermagem, pela UFSC. Docente da Universidade Estadual do Oeste do Paraná, campus Cascavel/PR e Universidade Paranaense/UNIPAR, campus Cascavel- PR. Brasil. E-mail: Maraschin@certto.com.br 


\section{Estudo da morbidade em uma população idosa}

\section{INTRODUÇÃO}

A constituição brasileira de 1988 ampliou os direitos dos cidadãos, provocando mudanças no papel do Estado e na estrutura jurídico-institucional do sistema público de saúde, designando novas relações entre as esferas Federal, Estadual e Municipal, indicando novos papéis entre os atores envolvidos, originando o Sistema Único de Saúde (SUS).

As Leis 8.080/90 e 8.142/90 indicaram princípios, diretrizes gerais e condições para a organização e funcionamento do sistema de saúde. Nessa perspectiva, a portaria 399/GM de 2006 estabeleceu o Pacto pela Saúde no que tange à consolidação do SUS e a redefinição das responsabilidades governamentais, bem como o compromisso entre os gestores no atendimento às prioridades que causam impacto sobre a situação de saúde da população brasileira. Em suma, propiciou a definição de prioridades articuladas e integradas em três componentes: Pacto pela Vida, Pacto em Defesa do SUS e Pacto de Gestão do SUS, salientando o Pacto pela Vida com previsão de diretrizes e ações estratégicas para a saúde do idoso. ${ }^{1}$

Observa-se no Brasil rápido envelhecimento populacional, resultante da redução das taxas de fecundidade, que vem ocorrendo desde os anos 60, e a diminuição progressiva das taxas de mortalidade a partir do final da segunda guerra mundial nos anos $40 .^{2}$ Com a mudança no perfil de saúde da população, tornam-se predominantes as doenças crônicas e suas complicações, que resultam na maior utilização dos serviços de saúde, ao invés de processos agudos que são resolvidos rapidamente, seja pela cura ou óbito. ${ }^{2}$ Cabe salientar que a morbidade ocupa lugar de destaque, principalmente para as doenças que não são disponibilizados mecanismos eficazes na atenção primária, secundária e terciária. 0 crescimento da população idosa será acompanhado por alteração no quadro de morbidade, consequência do aumento das doenças crônicodegenerativas e suas sequelas. ${ }^{2}$

Enquanto alguns idosos podem realizar atividades de vida diária (AVD), seu trabalho e seu lazer independente e serem saudáveis; outros, em decorrência de problemas de saúde, podem apresentar dependência para a realização destas atividades, ou ainda para tarefas instrumentais de vida diária que requeiram habilidades mais complexas. Proporcionalmente à população geral, o idoso costuma apresentar mais doenças, em geral crônicas, e o envelhecimento, quando acompanhado por doenças crônico-degenerativas com comprometimento da capacidade funcional, resulta em problemas de considerável

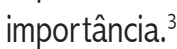

A perda da capacidade de função do idoso decorrente de doenças físicas ou mentais pode culminar na necessidade de intervenção e de adaptações para se manter as atividades rotineiras e o estilo de vida, as quais requerem trabalho constante dos profissionais que atuam, principalmente, na atenção básica.
Ações preventivas, assistenciais e de reabilitação devem objetivar a melhoria da capacidade funcional ou, no mínimo, a sua manutenção e, sempre que possível, a recuperação dela. Assim, o aumento da expectativa de vida da população amplia a demanda de idosos nos serviços de saúde, exigindo formação e capacitação no que tange ao atendimento de suas necessidades. ${ }^{4}$ Cabe destacar que os profissionais deverão estar preparados para identificar esses indivíduos, provendo-lhes uma assistência diferenciada.

0 envelhecimento populacional traz à tona o que é ser idoso. Para este estudo utilizou-se a definição que considera, nos países em desenvolvimento, idosos os indivíduos com idade igual ou superior a sessenta anos. ${ }^{5}$

A morbidade deve considerar a capacidade funcional como condição que determina sua real amplitude. A relação entre as doenças e a capacidade funcional remete à compreensão do conceito de saúde no processo de envelhecimento, pois a inexistência de doenças ou limitações é um fenômeno incomum.

A velhice é uma etapa do ciclo da vida e, portanto, está despertando interesse para os problemas enfrentados pelos idosos e expondo a necessidade de se garantir condições que favoreçam o envelhecimento com qualidade de vida. ${ }^{6}$

Desta forma, este estudo objetivou identificar os agravos que mais acometeram a população idosa no período de 1998 a 2005 e a capacidade funcional dos idosos residentes em área de abrangência de quatro unidades básicas de saúde, localizadas em diferentes regiões, no Município de Cascavel, Paraná, em 2007.

\section{MÉTODO}

No primeiro momento foi realizada uma pesquisa na base de dados do Sistema de Informações Hospitalar do Sistema Único de Saúde (SIH-SUS), para se verificar a morbidade prevalente no período de 1998 a 2005. Este sistema, criado em 1991, revela-se importante campo de investigação para análise epidemiológica, fonte de dados de morbidade respeitável pelo registro sistemático e abrangente. O SIH-SUS vem sendo considerado confiável e ferramenta útil para o monitoramento dos serviços de saúde. ${ }^{7}$

No segundo momento foi realizada a pesquisa de campo, utilizando um roteiro de entrevistas com questões fechadas e semiabertas. A construção das questões relativas à capacidade funcional foi baseada na escala de Atividades da Vida Diária de Katz e de Atividades Instrumentais de vida Diária de Lawton.

No que concerne à pesquisa de campo, a população estudada foi constituída por idosos de ambos os sexos, pessoas de 60 anos de idade ou mais, com capacidade de comunicação verbal, orientadas no tempo e no espaço e 
residentes em um dos bairros selecionados para a pesquisa, totalizando 141 sujeitos participantes do estudo.

A partir da projeção do censo de $2000,{ }^{8}$ identificouse, para o ano de 2006, o total de 17.717 idosos no município de Cascavel; destes, 2.478 (14\%) eram moradores na área de abrangência das UBS. Utilizando a fórmula $\mathrm{n}=\frac{\mathrm{Z}_{\alpha / 2}^{2}(1-\mathrm{p}) \mathrm{p}}{\mathrm{E}^{2}}$, na qual $Z_{\alpha / 2}^{2}$ corresponde ao nível de significância, $p=$ a proporção de idosos e $E=$ ao erro admitido, estabeleceu-se o número total de 141 idosos a serem entrevistados nas áreas de abrangência do estudo, que podem ser observados na Tabela $1 .{ }^{9}$

Tabela 1 - Distribuição de idosos por Unidade Básica de Saúde e amostra em 2006.

\begin{tabular}{lccc}
\hline \multicolumn{1}{c}{ UBS } & Total de idosos no ano 2000 & $\begin{array}{c}\text { Projeção pela taxa média de } \\
\text { idosos no ano 2006 }\end{array}$ & Amostra (n) \\
\hline Cascavel & 377 & 429 & 24 \\
Velho & & & 44 \\
Floresta & 680 & 773 & 28 \\
Guarujá & 431 & 490 & 45 \\
\hline Santa Cruz & 691 & 786 & 141 \\
\hline Total & 2.179 & 2.478 & \\
\hline
\end{tabular}

Fonte: IBGE (2006).

A técnica de amostragem foi a estratificada proporcional, que refere como um subconjunto de população (Ni) a qual somada gera o contingente total. Assim, no estudo proposto há quatro grupos ou extratos formados pelas quatro UBS, em que cada uma é homogênea internamente e heterogênea entre elas, inclusive na questão quantidade de indivíduos em cada grupo. ${ }^{9}$

0 município estudado está dividido administrativamente em dois distritos sanitários. A pesquisa foi realizada com os idosos residentes nas áreas de abrangência das Unidades Básicas de Saúde (UBS) do Bairro Guarujá, Santa Cruz, Cascavel Velho e Floresta. As duas primeiras unidades localizavam-se no distrito I e as duas últimas no distrito II, todas prestavam atendimento até às 22h00min.

Com o mapa do município foi possível identificar o limite geográfico de cada área de abrangência das UBS, e, por sorteio aleatório, selecionou-se a quantidade de quadras correspondentes ao número de idosos a serem entrevistados. Em cada quadra sorteava-se duas casas aleatoriamente, e a entrevista era realizada apenas com um idoso de cada residência.

Quando a casa selecionada não tinha nenhum idoso, seguia-se para a subsequente, em ordem crescente, até fechar a quadra correspondente. Quando no quarteirão selecionado não era encontrado idoso, seguia-se para o seguinte em ordem crescente ao número da casa.

Os dados foram coletados nos meses de maio a junho de 2007 e analisados de forma quantitativa, utilizando a estatística descritiva.

Obedecendo aos requisitos éticos de pesquisas envolvendo seres humanos, o projeto de pesquisa foi aprovado pelo Comitê de Ética em Pesquisa da Universidade Estadual de Maringá e aprovado pelo parecer $\mathrm{N}^{0}$. 033/2007.

\section{RESULTADOS E DISCUSSÕES}

\section{As internações dos idosos em Cascavel}

No Brasil, em 1998, as cinco primeiras causas de internamentos foram: doenças do aparelho circulatório (IX), doenças do aparelho respiratório $(X)$, doenças do aparelho digestivo (XI), doenças do aparelho geniturinário (XIV) e algumas doenças infecciosas e parasitárias (I). No ano de 2005 as causas de internamento foram praticamente as mesmas, com alteração apenas na $4^{\text {a }}$ causa com o registro das neoplasias (II) e não mais o das doenças do aparelho geniturinário. ${ }^{10}$

No Paraná, as cinco primeiras causas de internamento em 1998 foram as mesmas assinaladas acima, em âmbito nacional. A ordem de ocorrência também pouco se alterou em 2005 e, da mesma forma, o aparecimento das neoplasias como $4^{\text {a }}$ causa registrada ${ }^{10}$.

0 Gráfico 1 mostra que em Cascavel as doenças do aparelho circulatório, também foram as responsáveis pelo maior número de internamento entre os idosos no período de 1998 a 2005. Ainda com relação a este tipo de internamento, a diminuição mais relevante do número de casos ocorreu de 1998 para 1999 com 262 ocorrências, e em 2001 para 2002, com 65 casos. Dentre as cinco causas de morbidade, o maior número foi registrado em 2003 com 1.054 casos pelas doenças do aparelho circulatório ${ }^{10}$.

Deve-se considerar que o quadro de morbidade, aqui apresentado, diz respeito somente às internações pagas pelo SUS com a Autorização de Internamento Hospitalar (AlH/SUS). Não foram computadas as internações particulares e de convênios, pois a Comunicação de Internação Hospitalar $(\mathrm{ClH})$ não tinha regularidade na apresentação dos dados. 
Gráfico 1 - Internaç̧ões hospitalares de idosos, segundo grupos de causa da doença (CID 10), Cascavel, PR de 1998 a 2005.

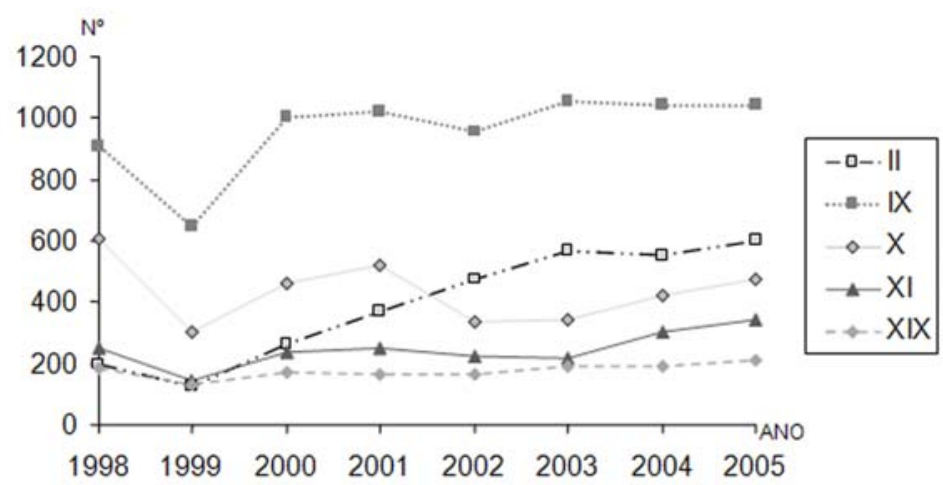

Fonte: DATASUS - Movimento de AlH - Arquivos reduzidos - Brasil 2006.

Comparando-se os dados de Cascavel no ano de 1998 com o do Estado do Paraná e do Brasil no mesmo ano, verificouse que as causas de internamentos foram semelhantes, com exceção das doenças geniturinárias, das lesões, envenenamento e algumas outras consequências de causas externas e doenças infecciosas e parasitárias.

No ano de 2005, as doenças do aparelho circulatório continuaram aparecendo como principal causa de morbidade hospitalar no município, no estado e no país. Porém, a quinta causa de morbidade no estado e no país corresponderam às doenças infecciosas e parasitárias. No município, foram as lesões, envenenamento e algumas outras consequências de causas externas.

Estudos populacionais realizados com a pessoa idosa no Brasil demonstram que a situação é similar e ocorre em todas as regiões, variando, entretanto, o valor das taxas. Quanto às internações pagas pelo SUS às pessoas de 60 anos ou mais, as doenças do aparelho circulatório tem destaque no total de hospitalizações. As doenças do aparelho respiratório ocuparam o segundo lugar, sendo seguidas das internações por doenças do aparelho digestivo. ${ }^{11}$

Em suma, no município estudado, os capítulos da CID 10 que mais levaram os idosos à hospitalização durante o período analisado foram as doenças relacionadas ao aparelho circulatório, respiratório, neoplasias, digestivo, lesões, envenenamento e algumas outras consequências de causas externas, variando a classificação das mesmas durante os anos analisados.

\section{0 que disseram os idosos}

É necessário pontuar que as Doenças do Aparelho Circulatório foram as responsáveis pelo maior número de doenças referidas pelos idosos, assim como as principais causas de morbimortalidade no município.

Nas entrevistas realizadas com os idosos, os mesmos relataram, ainda, outras doenças tais como:
Catarata, úlcera gástrica, gastrite, hérnia, alergia, rinite, espondilite crônica, hemorroida, labirintite, fraqueza, refluxo gástrico, epilepsia, trombose, doença dermatológica, hipotensão, problema de estômago, insuficiência respiratória, problemas de vesícula e varizes.

Com relação às doenças referidas, nota-se que 102 $(73 \%)$ entrevistados declararam sofrer de uma a três doenças e 12,1\%, de quatro a sete. A média de enfermidades referida pelos idosos foi de: 1,5 na UBS Cascavel Velho; 2,4 na UBS Floresta; 2,1 na UBS do Guarujá e 2,2 na UBS Santa Cruz. A média geral foi de 2,1 doenças. Do total dos idosos pesquisados, $21(14.9 \%)$ afirmaram não possuir qualquer doença; destes, $12(57,1 \%)$ estavam acima de 70 anos.

Mesmo com a média apresentada de duas doenças por entrevistado, nove deles $(6,4 \%)$ referiram que sua saúde era muito boa. Ainda $42(29,8 \%)$ disseram que era boa; 74 (52,5\%), regular; $15(10,6 \%)$ consideraram-na ruim e apenas um referiu ser muito ruim; este estava com 84 anos e morava em domicílio multigeracional. Para os que consideram seu estado de saúde ruim, a maioria apontou como causa a hipertensão arterial e doença da coluna ou costas.

Dentre os idosos que citaram seu estado de saúde como regular, 49,7\% disseram ser acometidos por uma ou mais patologias. Esses dados estão em concordância com um estudo realizado no município de São Paulo em que uma proporção elevada dos entrevistados declararam ter três ou mais doenças, sendo o fato registrado entre $48 \%$ das mulheres e $33 \%$ dos homens. A presença de pelo menos uma doença crônica pode significar maior utilização dos serviços de saúde. ${ }^{12}$

Outra questão levantada denota que dez idosos $(7,1 \%)$ estiveram acamados nos últimos 15 dias; quatro com mais de 75 anos e três com idade entre 65 a 69 anos. 0 tempo no leito variou de 1 a 14 dias, apenas uma idosa de 109 anos apresentava-se acamada há 5 meses. 
As doenças mais citadas pelos idosos entrevistados, considerando todas as faixas etárias, nas UBSs pesquisadas foram:

Hipertensão arterial, doença de coluna ou costas, doença do coração, diabetes e artrite ou reumatismo.

A hipertensão foi mais referida pelas idosas, nos Bairros Cascavel Velho, Floresta e Santa Cruz, apresentando uma relação média de quatro mulheres para um homem portador de hipertensão arterial. No Bairro Guarujá, a distribuição foi mais equilibrada; dos 13 que referiram a doença, oito eram senhoras, e a maioria das mulheres concentrava-se em faixas etárias mais elevadas que a dos homens.

Quanto ao sexo feminino ser mais acometido pela hipertensão arterial, lembra-se que até os 40 anos, os níveis de pressão arterial apresentam-se mais elevados nos homens, mas, a partir desta faixa etária, as mulheres passam a ser mais acometidas devido à instalação do período do climatério com a consequente perda da proteção dos hormônios femininos sobre o sistema cardiovascular. ${ }^{13}$

Outro aspecto que merece destaque refere-se a crescente expectativa de vida que não ocorre de modo uniforme em ambos os sexos. As diferenças foram encontradas em razão de situações como: exposição às causas de risco de trabalho (menor repercussão dos fatores de risco em relação ao homem); maior incidência de morte por causas externas (acidentes em geral, acidentes de trânsito, homicídios, quedas, suicídios e outras), sendo estas quatro vezes superiores no homem; em função das diferenças no consumo do tabaco e álcool, produtos que estão associados às causas de mortes mais importantes como as doenças cardiovasculares. ${ }^{2-13}$

Destaca-se também a diferença de atitude quanto às doenças e incapacidades. ${ }^{2}$ As mulheres tem melhor conhecimento das doenças e em geral, estão mais atentas ao aparecimento de sintomas, utilizando mais os serviços de saúde do que os homens. Uma das causas de melhor prognóstico das doenças crônicas é a procura precoce pela assistência médica nesse grupo populacional.

No entanto, os fatores de risco predominantes no homem, e que reduzem o seu período de vida, muito em breve podem estar acometendo as mulheres devido a sua mudança de hábitos de vida, decorrentes da ampliação do seu espaço no mundo contemporâneo. ${ }^{14}$

Um fato que chama atenção no período de 1998 a $2005^{10}$ é o número de mortes, que entre os homens é maior, excetuando-se as doenças endócrinas, nutricionais e metabólicas quando se observa maior registro entre as mulheres. A situação encontrada é a mesma para as internações, ou seja, os homens representaram a maioria de idosos internados no período, somando 11.570 (51\%) dos internamentos, contra $11.115(49 \%)$ das mulheres. ${ }^{10}$ Isso pode significar a necessidade do incremento das ações e serviços de saúde focados no homem. Para operacionalizála há que se implantar na rede básica novas rotinas em programas preventivos, educativos, melhoria no diagnóstico e tratamento precoce.

Por esse prisma, a equipe de saúde necessita de uma interação de saberes e práticas, necessárias para o cuidado integral à saúde dos idosos.

Tabela 2 - Distribuição das doenças referidas e que mais incomodavam, por grupo de causa e por Unidade Básica de Saúde do município de Cascavel no ano de 2007.

\begin{tabular}{|c|c|c|c|c|c|c|c|c|}
\hline \multirow[t]{2}{*}{ Grupo de causas } & \multicolumn{8}{|c|}{ Unidades Básicas de Saúde } \\
\hline & $\mathrm{CV}$ & $\%$ & $\mathrm{~F}$ & $\%$ & G & $\%$ & $\mathrm{SC}$ & $\%$ \\
\hline \multicolumn{9}{|l|}{ Doença referida } \\
\hline Doenças do Aparelho Circulatório (IX) & 19 & 13,5 & 38 & 27,0 & 19 & 13,5 & 35 & 24,8 \\
\hline Doenças do Sistema Osteomuscular e do Tecido Conjuntivo (XIII) & 8 & 5,7 & 25 & 17,7 & 15 & 10,7 & 24 & 17,0 \\
\hline Doenças Endócrinas Nutricionais e Metabólicas (M) & 3 & 2,0 & 14 & 9,9 & 9 & 6,4 & 9 & 6,4 \\
\hline Doenças do Aparelho Respiratório $(X)$ & 2 & 1,4 & 3 & 2,0 & 3 & 2,0 & 6 & 4,3 \\
\hline Doenças do Aparelho Diqestivo (XI) & 1 & 0,7 & 6 & 4,3 & 3 & 2,0 & 3 & 2,0 \\
\hline \multicolumn{9}{|l|}{ Doença que mais incomoda } \\
\hline Doenças do Sistema Osteomuscular e do Tecido Conjuntivo (XIII) & 3 & 2,0 & 16 & 11,4 & 11 & 7,8 & 10 & 7,1 \\
\hline Doenças do Aparelho Circulatório (X) & 9 & 6.4 & 3 & 2,0 & 3 & 2,0 & 12 & 8,5 \\
\hline Doenças do Apar elho Respiratório $(X)$ & 1 & 0,7 & 2 & 1,4 & 3 & 2,0 & 3 & 2,0 \\
\hline Doenças Endócrina, Nutricionais e Metabólicas (IV) & - & - & 3 & 2,0 & 4 & 2,7 & 1 & 0,7 \\
\hline Doenças do Olho e Anexos (VII) & - & - & 4 & 2,7 & 3 & 2,0 & - & \\
\hline
\end{tabular}

Fonte: Entrevista com os idosos 


\section{Estudo da morbidade em uma população idosa}

Destaca-se que a Tabela 2 foi agrupada conforme a classificação estatística internacional de doenças e problemas relacionados à saúde (CID 10) por capítulo, tanto as doenças referidas como as que mais incomodam os idosos.

Quando condensados na Tabela 2 os registros das entrevistas com os idosos, verifica-se a ocorrência das doenças referidas da $1^{\text {a }}$ a $5^{\text {a }}$ causa, que, respectivamente, foram as do aparelho circulatório (IX), com o mesmo comportamento tanto no bairro do Cascavel velho como no Guarujá e maior ocorrência nos bairros Floresta e Santa Cruz. As doenças do sistema osteomuscular e do tecido conjuntivo (XIII) mostram desempenho equivalentes nos bairros Floresta e Santa Cruz, com menor ocorrência nos bairros Guarujá e Cascavel Velho. As doenças endócrinas nutricionais e metabólicas (IV) tem maior ocorrência nos bairros Floresta, Guarujá e Santa Cruz, enquanto as doenças do aparelho respiratório $(X)$ apresentam maior ocorrência no bairro Santa Cruz e as doenças do aparelho digestivo (XI), maior ocorrência no bairro Floresta.

Tanto para as doenças referidas quanto para as que mais incomodam os idosos, o comparativo de dados da $1^{\mathrm{a}}$ a $4^{\circ}$ causa apresentadas é o mesmo. Há inversão apenas entre a $5^{a}$ causa, que os idosos relatam serem as doenças do aparelho digestivo (XI), enquanto as que mais incomodam são as doenças do olho e anexos (VII).

Entre as doenças referidas registrou-se a ocorrência das doenças do sistema osteomuscular e do tecido conjuntivo como $2^{\mathrm{a}}$ causa, seguidas pelas doenças endócrinas nutricionais e metabólicas, que não foram registradas entre as primeiras ocorrências de internamento em 1998 e nem em 2005.

Ressalta-se que, entre as doenças do sistema osteomuscular e do tecido conjuntivo, foram citadas pelos idosos:

Osteoporose, artrose, espondilite crônica e artrite ou reumatismo, doença de coluna ou costas.

Estas doenças estão aumentando rapidamente entre os idosos, tornando-se um problema de saúde pública.

Os idosos constituem um grupo populacional de risco para as doenças do sistema osteomuscular e do tecido conjuntivo e, portanto, merecem abordagem especial dos programas de controle da doença, que devem levar em consideração as peculiaridades desta faixa etária. As equipes multiprofissionais das unidades básicas de saúde são importantes para a reversão desse quadro. Elas devem estar capacitadas e instrumentalizadas para poder educar a comunidade em que atuam, podendo assim fazer diagnóstico rápido e preciso, instituir o tratamento e acompanhamento adequado.

As alterações da classificação entre as doenças referidas e as que mais incomodavam permitiram identificar a prevalência de doenças crônicas nessa população idosa. Esses dados ajudam na compreensão das mudanças que vêm ocorrendo no processo de envelhecimento e alertam para os possíveis impactos negativos, e, embora haja uma preocupação com a terceira idade, ainda são incipientes as alternativas e soluções que melhorem essa fase da vida.

Destacam-se como a $5^{\text {a }}$ causa, que mais incomoda os idosos, as doenças do olho e anexos, dentre as quais foram citadas:

Cegueira, baixa acuidade visual, degeneração irreversível da retina, dor nos olhos e catarata.

Assinala-se a demanda crescente das doenças do sistema osteomuscular e do tecido conjuntivo, bem como doenças do olho e anexos não figuram entre as principais causas de morte e internação, permanecendo invisível para a saúde pública.

Lembrando que, entre os distúrbios oftálmicos, a cegueira é a incapacidade mais temida pelas pessoas idosas, causando impacto negativo em sua qualidade de vida. Salientase que os médicos que tratam de pacientes geriátricos têm a responsabilidade do diagnóstico precoce. ${ }^{14}$

Os idosos apresentam menos doenças agudas em relação aos adultos jovens, mas, quando são acometidos por um quadro agudo, eles comumente requerem extensos períodos de recuperação e desenvolvem mais complicações que as pessoas mais jovens. ${ }^{15}$

A principal preocupação para o idoso é a doença crônica, representada pela enfermidade de longa duração que mostra pouca alteração. Dessa forma, as doenças crônicas, também chamadas de doença crônica não transmissível (DCNT), têm mais probabilidade de desenvolver uma incapacidade, e o desafio é combater os efeitos das DCNT nos idosos, para que eles atinjam sua máxima capacidade funcional. ${ }^{15}$

Salienta-se que com o aumento da expectativa de vida da população, cresce a incidência das DCNT que fragilizam o idoso, gerando maior dependência e menor autonomia para a realização das suas atividades diárias. ${ }^{4}$

Com a aplicação da Escala de Atividades Básicas de Vida Diária, foi possível verificar que os entrevistados, de maneira geral, mostraram-se independentes, pois 136 $(96,5 \%)$ afirmaram conseguir realizar todas as atividades cotidianas, sem o auxílio de outras pessoas; três (2,1\%) referiram dependência parcial, com necessidade de ajuda para o desempenho de alguma atividade, e dois $(1,4 \%)$ apresentavam dependência importante para o controle dos esfíncteres, banhar-se, vestir-se e movimentar-se.

Os idosos com dependência parcial estavam com idade acima de 75 anos e informaram padecer de uma ou mais doença crônica. Um dos idosos que apresentava dependência importante tinha 109 anos; outro com 90 anos possuía sequela de AVC, e ambos referiram falta de visão e fraqueza. 0 declínio natural do processo de envelhecimento, muitas vezes, é acompanhado pela diminuição da capacidade funcional do organismo. Para os autores, ${ }^{2-15} \mathrm{a}$ 
velocidade desse declínio exibe variabilidade, depende de fatores genéticos, hábitos e estilos de vida, contexto socioeconômico e cultural, entre outros.

Tanto as pessoas com dependência parcial como as com dependência importante irão aumentar a necessidade de cuidados, seja formal ou informal. A evolução da dependência, como processo dinâmico, pode se modificar e até ser prevenida ou reduzida se houver ambiente e assistência adequados. ${ }^{16}$

No que diz respeito às Atividades Instrumentais de Vida Diária, podem-se observar no Gráfico 2 as atividades que os idosos: realizam sem ajuda, com ajuda parcial ou não conseguem realizar.

Gráfico 2 - Distribuição dos idosos, conforme escala de Lawton para Atividades Instrumentais de Vida Diária no ano de 2007.

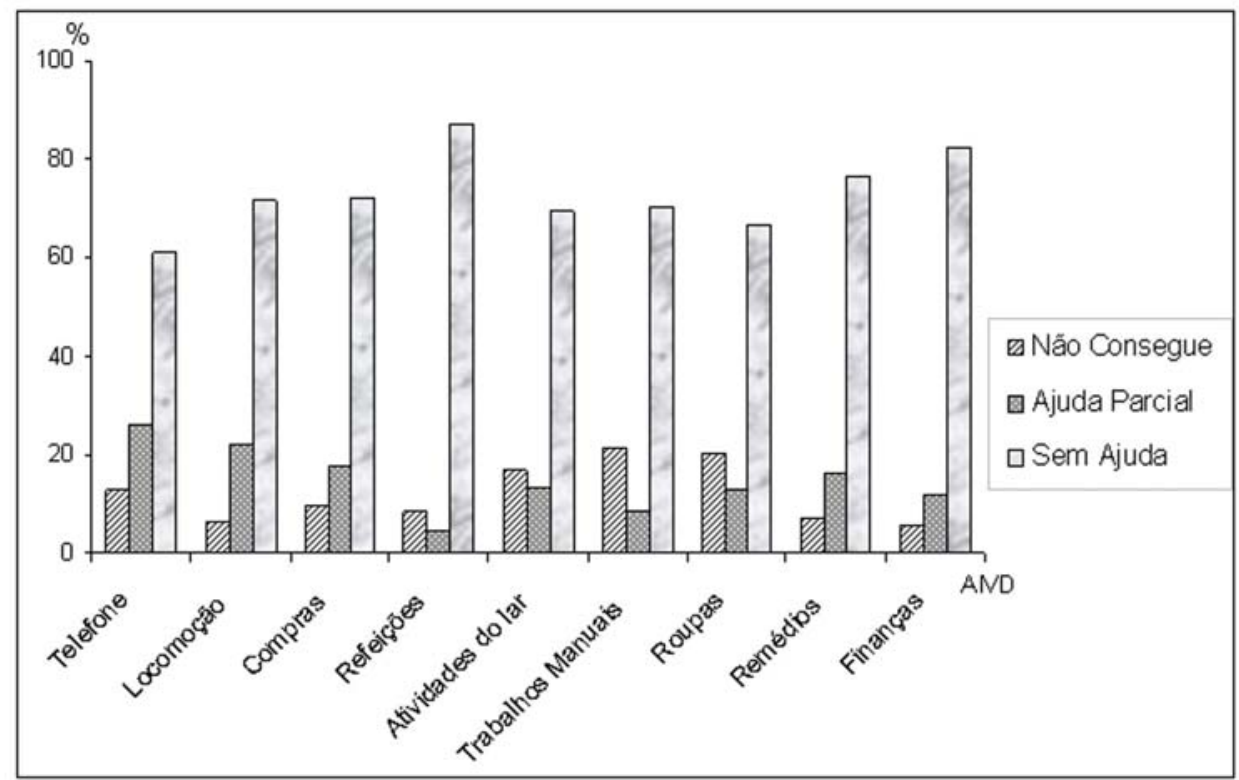

Fonte: Entrevista com os idosos.

Tanto para AVD como AIVD, os idosos foram classificados como independentes funcionais com capacidade de realizar atividades sem ajuda, mas não se podem ignorar os idosos com dependência parcial e dependência importante, pois a dificuldade para desempenhar tarefas de cuidado pessoal e precisar de outros para completá-las pode ter fundamental importância no bem-estar e na autoestima do idoso. Deve-se lembrar que as políticas sociais voltadas para os idosos tem-se organizado de forma fragmentada e ao atendimento individualizado, desconsiderando a família e a comunidade. ${ }^{17}$ Todas as escalas que medem a capacidade funcional auxiliam na classificação e adequação dos processos terapêuticos e também podem propor programas governamentais de apoio aos idosos.

0 sistema de saúde terá que enfrentar essa crescente demanda por procedimentos diagnósticos e terapêuticos das DCNT, bem como uma demanda maior por serviços de reabilitação. Destaca-se que será necessário estabelecer indicadores de saúde capazes de identificar o idoso de alto risco de perda funcional e orientar ações de promoção de saúde e manutenção da capacidade funcional. ${ }^{18}$

As limitações e/ou restrições de capacidade funcional têm importância no âmbito das consequências dos agravos das doenças crônicas, mas a presença da doença não significa que o idoso não possa gerir sua própria vida e viver o seu cotidiano de forma independente. ${ }^{18}$ Usualmente as limitações e/ou restrições das atividades habituais nos idosos podem evoluir para a deficiência, incapacidade e desvantagens.

Foi perguntado aos idosos sobre quem havia the informado sobre a patologia referida. Para a maioria $(82,3 \%)$ dos entrevistados, o diagnóstico foi feito pelo médico; 0,7\% pela pastoral da saúde; 2,1\% autorreferenciado, e 21 (15\%) não citaram doenças. A princípio esses dados configuram melhoria do acesso aos serviços de saúde, revelado pelo elevado número de idosos que foram diagnosticados pelos médicos. Este fato pode estar associado à ampliação das UBS e, consequentemente, ao acesso dos serviços de saúde. Será que antes da ampliação das unidades básicas de saúde os percentuais dos resultados seriam os mesmos?

Verificou-se haver consistência nos resultados com os do estudo realizado, em sete cidades da América Latina, no inquérito domiciliar com os idosos de ambos os sexos, identificando prevalência semelhante entre as doenças referidas, bem como 0 fato de haver sido o médico o principal informante. ${ }^{12}$

\section{CONCLUSÕES}

Cabe dizer que o tema proposto foi estimulante, pois o estudo do envelhecimento humano é pertinente na atualidade e espera-se que seja oportuno para o município, tendo em vista 
o momento favorável na atenção à saúde do idoso, conforme recomendação do Ministério da Saúde, aos gestores, no que se refere ao atendimento, e às prioridades que causem impacto sobre a situação de saúde dessa população.

As entrevistas com os idosos transcorreram de forma simples e fácil, pois os sujeitos mostraram-se disponíveis e dispostos em colaborar. Já a coleta dos dados secundários nas bases de dados disponíveis no município foi mais complicada. A dificuldade encontrada estava relacionada à divergência de informações sobre a população adscrita em cada UBS.

Conforme os resultados obtidos neste trabalho, devido ao aumento da expectativa de vida da população, o envelhecimento se destaca tanto no município de Cascavel como no estado e país, e é um desafio na atualidade para as três esferas de governo. Por isso, é necessário implantar serviços voltados para as doenças crônicas, com foco na manutenção da máxima capacidade funcional pelo maior tempo possível, valorizando a autonomia e a autodeterminação dos idosos.

0 desafio para as políticas públicas está em ajustar a assistência à saúde para atender o emergente contingente de idosos, na morbidade que se apresenta ascendente, principalmente para as Doenças do Aparelho Circulatório, as Neoplasias, as Doenças do Aparelho Respiratório e as Endócrinas, Nutricionais e Metabólicas. Outro aspecto relevante da morbidade foram as Doenças do Sistema Osteomuscular, do Tecido Conjuntivo e as dos Olhos e Anexos, referidas pelos entrevistados.

Diante disso, percebe-se a necessidade de educação e ações multidisciplinares como uma das principais estratégias para o controle da morbidade e mortalidade, proporcionando condições diferenciadas, iniciando pela sensibilização e responsabilização dos profissionais acerca do seu papel junto à sociedade. Deve-se trabalhar na prevenção, promovendo ações individuais e em grupo, ações educativas, trazendo-os cuidadosamente para uma realidade que inclua atitudes saudáveis e preventivas que o conduzam a uma melhor qualidade de vida.

A luta pela efetiva assistência à saúde do idoso, na perspectiva de um sistema público de saúde articulado, integrado e comprometido, ainda é uma meta a ser conquistada.

\section{REFERÊNCIAS}

1. Ministério da Saúde (BR). Secretaria Executiva. Departamento de Apoio à Descentralização. Diretrizes operacionais dos pactos pela vida, em defesa do SUS e de gestão. Brasília (DF); 2006. v.1.

2. Chaimowicz F. Epidemiologia e o envelhecimento no Brasil. In: Freitas EV, et al. Tratado de geriatria e gerontologia. $2^{\mathrm{a}}$ ed. Rio de Janeiro: Guanabara Koogan; 2006. p.106-130.

3. Tirado MGA. Reabilitação e manutenção da capacidade funcional. In: Saldanha AL, Caldas CP, organizadores. Saúde do idoso: a arte de cuidar. $2^{a}$ ed. Rio de Janeiro: Interciência; 2004. p.144-51.

4. Paes PFA, Espírito Santo FH. Limites e possibilidades no cotidiano do familiar que cuida do idoso com Alzheimer no ambiente domiciliar. Esc Anna Nery. 2005 ago; 9 (2): 192-98.

5. Lei $n^{0} 10741$, de 01 de outubro de 2003. Dispôe sobre o Estatuto do Idoso e dá outras providências. $2^{\mathrm{a}}$ ed. Brasília (DF); 2008. (Série E: Legislação do Brasil)

6. Assis M. 0 envelhecimento e suas conseqüências. In: Caldas CP, organizadora. A saúde do idoso: a arte de cuidar. Rio de Janeiro: EdUERJ; 1998. p.39-48.

7. Bittencourt AS, Camacho LAB, Leal MC. O Sistema de Informação hospitalar e sua aplicação na saúde coletiva. Cad Saude Publica. 2006 jan; 22(1): 19-30.

8. Instituto Brasileiro de Geografia e Estatística - IBGE [on-line]. Censo populacional 1991, 2000 e contagem populacional de 1996. Brasília(DF); 2000. [citado 2006]. Disponível em: http:// www.saude.pr.gov.br/Estatisticas/pop/index.html.

9. Freund JE, Simon GA. Estatística aplicada. Tradução de Alfredo Alves de Farias. 9ª ed. Porto Alegre: Bookman; 2000.

10. Ministério da Saúde(BR). Departamento de Informática do SUS. Informações de Saúde. Assistência à saúde. Internações hospitalares [on-line]. [citado 2006]. Disponível em: http://tabnet.datasus.gov.br/ cgi/deftohtm.exe?sih/cnv/rxpr.def.

11. Côrte B, Oliveira B, Medeiros S. Brasil: o que dizem os números sobre a pessoa idosa? [on-line]. [citado 2007]. Disponível em: http:/ /www.abep.nepo.unicamp.br/encontro2006/ocspdf/ ABEP2006_190.pdf.

12. Lebrão ML, Laurenti R. Condições de saúde. In: Saúde, bem-estar e envelhecimento. 0 Projeto SABE no município de São Paulo: uma abordagem inicial. Brasília (DF): Organização Pan-Americana de Saúde; 2003. p.75-91.

13. Monteiro PC, Santos FS, Fornazari PA, Cesarino CB. Características biossociais, hábitos de vida e controle da pressão arterial dos pacientes em um programa de hipertensão. Arq Cienc Saude. 2005 abr/jun; 12 (2): 73-79.

14. Kollarits CR. Distúrbios oftálmicos. In: Dutbie Júnior EH, Katz PR. Geriatria prática. $3^{\mathrm{a}}$ ed. Rio de Janeiro (RJ): Revinter; 2002. p.44958.

15. Roach S. Introdução à enfermagem gerontológica. Tradução de Ivone Evangelista Cabral, Marcia Tereza Luz Lisboa. Rio de Janeiro (RJ): Guanabara Koogan; 2003.

16. Caldas CP. Envelhecimento com dependência: responsabilidades e demandas da família. Cad Saude Publica 2003 maio/jun; 19 (3): 77381. 
17. Farinasso ALC, Marques S, Rodrigues RAP, Haas VJ. Capacidade funcional e morbidades referidas de idosos em uma área de abrangência do PSF. Rev Gaucha Enferm. 2006 mar; 27(1): 45-52.

18. Ramos LR. A mudança de paradigma na saúde e o conceito de capacidade funcional. In: Guia de geriatria e gerontologia. Barueri: Manole; 2005. p.1-7. 